\title{
Análise da evolução do preenchimento de prontuários clínicos institucionais com a implantação de uma gestão de risco
}

\author{
Karyn Sabrina Marinho Umbelino*; Natália de Souza Silva**; Márcia Cristina da Silva***; Fernanda \\ do Nascimento de Lemos Campos****; Luiz Fernando Lolli*****
}

\author{
* Cirurgiã-dentista egressa da Universidade Estadual de \\ Maringá \\ ** Graduanda em Odontologia, Universidade Estadual de \\ Maringá \\ *** Residente, Programa de Saúde Coletiva e da Família, \\ Universidade Estadual de Maringá \\ **** Mestranda, Programa de Pós-Graduação em Odontologia \\ Integrada, Universidade Estadual de Maringá \\ ****** Docente, Coordenador do Curso de Odontologia, \\ Universidade Estadual de Maringá
}

Recebido em 17/06/2019. Aprovado em 28/01/2020.

\begin{abstract}
RESUMO
Este estudo documental, longitudinal e quantitativo foi realizado com o objetivo de avaliar a evolução documental dos prontuários clínicos institucionais após a implantação da gestão de risco. No ano de 2017, o Grupo de Estudos de Orientação Profissional e Odontologia Legal (GEOPOL), da Universidade Estadual de Maringá (UEM) com o intuito de melhorar a produção documental e reduzir riscos. A presente pesquisa considerou 660 prontuários analisados pelo GEOPOL (Análise 1) no período de março de 2017 a abril de 2018. Entre maio e setembro de 2018, foram avaliados 422 prontuários (Análise 2), após a implantação da gestão de risco. Ambas as análises tiveram por base um check-list exclusivo, desenvolvido pelo GEOPOL para esta finalidade. Os dados foram processados em planilhas eletrônicas e os resultados foram apresentados de modo descritivo e analítico. Os resultados demonstraram que, no geral, as variáveis apresentaram um preenchimento melhor na Análise 2, demonstrando eficácia nos processos de gestão do GEOPOL. Conclui-se que houve evolução no preenchimento documental dos prontuários clínicos após a implantação da gestão de risco.
\end{abstract}

Descritores: Odontologia Legal. Documentação. Ética Odontológica. Gestão de Riscos. 


\section{INTRODUÇÃO}

Os prontuários clínicos odontológicos são documentos presentes em Instituições de Ensino Superior privadas ou públicas, elaborados por profissionais ou estudantes de graduação ou de pósgraduação, assim como em consultórios, clínicas privadas e públicas. Eles são de suma importância em termos de gestão clínica, administrativa e legal, representando os registros de informações dos pacientes que estão em atendimento ou que já foram atendidos ${ }^{1}$, sendo constituído então por um conjunto de termos, formulários e exames complementares, como tomografias, radiografias e ultrassonografias ${ }^{2-4}$.

Nos últimos anos, o tema "responsabilidade profissional" tem sido mais debatido na Odontologia, muito em função do aumento no número de ações judiciais e éticas contra os cirurgiões-dentistas (CDs), visto que os pacientes têm se apresentado mais exigentes e conscientes de seus direitos. Os CDs devem atentar para a prática denominada Odontologia Defensiva, que abrange o conjunto de medidas que visam prevenir a citação do profissional de Odontologia como agente em processos judiciais. Por essa razão, é muito importante tomar medidas de segurança para se prevenir de possíveis alegações de pacientes, e isso só é possível com o correto preenchimento e arquivamento desses documentos ${ }^{5}$.

É notável que diante da intensa rotina clínica odontológica, a manutenção da documentação que se refere ao paciente e ao tratamento em si, está revestida de muitos aspectos éticos e legais, além do que, a formação profissional em Odontologia deve envolver múltiplos aspectos relacionados à execução de ações clínicas somados ao desenvolvimento de competências organizacionais, gerenciais, éticas e legais, especialmente no que se refere à relação profissional-paciente ${ }^{3}$.

Os procedimentos odontológicos devem ser cuidadosamente registrados na documentação, com dados relacionados ao paciente que sejam pertinentes ao tratamento, como por exemplo sua identificação, história de saúde geral e bucal, exame físico, exames complementares, cópias de documentos emitidos, condições pré-existentes ao tratamento e qualquer outra informação fundamental ao manejo do usuário ${ }^{4}$.

O prontuário deve ser bem elaborado, atender ao aspecto ético-jurídico, mas também os aspectos clínicos, administrativos e odontolegais ${ }^{6}$. $\mathrm{O}$ aspecto jurídico tem mais relação com proteger o profissional de alegações no âmbito judicial, ético e administrativo. Contudo, ele deve servir para o acompanhamento da evolução clínica do paciente, possibilitar a gestão do empreendimento odontológico e servir de anteparo, com conteúdo probatório para os processos de identificação humana ${ }^{7}$.

No ano 2013 foi realizado um trabalho de conclusão $^{7}$ no Curso de Odontologia da Universidade Estadual de Maringá (UEM) que demonstrou a necessidade de aprimoramento no preenchimento dos documentos institucionais da Clínica Odontológica frente aos aspectos legais e éticos. Neste mesmo ano, também foram iniciadas as atividades do Grupo de Estudos de Orientação Profissional e Odontologia Legal (GEOPOL), presente no diretório de grupos do $\mathrm{CNPq}$ e institucionalizado na UEM como projeto de Ensino. Apesar da institucionalização como projeto de ensino, o GEOPOL atua hoje na tríade universitária, trabalhando aspectos de Ensino, Pesquisa e Extensão de forma interligada.

Tendo por base o referido estudo de 2013 e também para aprimorar a lógica do ensino e a segurança jurídica institucional, no ano de 2017 o GEOPOL propôs a Gestão de Risco Documental na Clínica Odontológica da UEM (COD-UEM). A gestão inclui o processamento documental da clínica, com revisão, aprimoramento e edição das normas institucionais, revisão, aprimoramento e edição do prontuário único, gestão de retirada de 
documentos por pacientes, gestão de preenchimento, melhoramento de arquivo e outros. Atuam no GEOPOL acadêmicos de graduação, pós-graduação lato sensu e stricto sensu, coordenados por um mediador, docente da área de Odontologia Legal.

No ambiente acadêmico, a correta elaboração da documentação é ainda mais crucial, por se tratar de um ambiente de aprendizado. Partindo desta premissa, foi desenvolvido um padrão de análise documental original e exclusivo do GEOPOL, com base nos aspectos legais e éticos da profissão odontológica e com ênfase na Odontologia Defensiva, que pudesse fazer uma classificação de risco nos documentos institucionais. A gestão de risco foi implantada, está em operacionalização e deve ser constantemente avaliada.

Considerando o exposto, este estudo teve por objetivo avaliar a evolução documental dos prontuários institucionais da COD-UEM após a implantação da gestão de risco documental.

\section{METODOLOGIA}

Trata-se de um estudo documental, longitudinal e quantitativo que avalia uma ação interventiva realizada sobre documentos odontológicos.

Para a amostra do estudo foram considerados os prontuários avaliados pelo GEOPOL, incluídos segundo os seguintes critérios: prontuários avaliados no todo, arquivados no diário de ocorrência do GEOPOL e produzidos apenas por acadêmicos de graduação em Odontologia da instituição pesquisada.

Aqueles que contemplavam os critérios acima descritos e analisados entre março de 2017 e abril de 2018 constituíram a amostra denominada "análise 1". Ao todo foram 660 prontuários nesta amostra.

Foi denominada de "análise 2", a amostra constituída por prontuários que tiveram registros de atendimentos entre maio e setembro de 2018. Esta amostra foi de 422 prontuários. A diferença amostral ocorreu porque em 238 prontuários não houve atendimento adicional registrado no relatório de atividades.

As variáveis consideradas foram agrupadas nas categorias: identificação e ciência do tratamento; anamnese e exames; assinaturas e consentimento e documentos emitidos.

Quanto à identificação e ciência do tratamento, considerou-se as seguintes variáveis: "Nome", se o nome do paciente estava presente e preenchido corretamente; "Documento", se havia registro do número de documento de identificação do paciente ou de seu responsável; "Assinatura”, se havia assinatura do paciente ou de seu responsável na autorização do tratamento; e "Termo de ciência", se estava presente e assinado.

Em relação às variáveis agrupadas como "anamnese e exames" foram observadas: "Anamnese", se as informações a respeito do paciente estavam preenchidas corretamente; "Exame físico", se foram realizados exames intra e extrabucais; "Odontograma", se o mesmo estava preenchido corretamente e à caneta; "Periograma", se estava preenchido corretamente e à caneta; "Plano de tratamento", se havia pelo menos duas opções terapêuticas para o paciente, preenchidas corretamente e à caneta.

Sobre assinaturas / consentimento analisouse: "Assinatura do Estudante", "Assinatura do Docente" e "Assinatura do Paciente" se havia a assinatura do estudante, docente e paciente respectivamente - no Plano de Tratamento escolhido.

As assinaturas no registro de procedimentos, consideraram "Assinatura do Estudante", "Assinatura do Docente" e "Assinatura do Paciente" quando havia a assinatura do estudante, docente e paciente - respectivamente - em todos os procedimentos realizados.

Sobre os documentos emitidos a favor do 
paciente e exames de imagem, ponderou-se "Atestados", se havia assinatura do paciente ou responsável na segunda via presente; "Receitas", se havia assinatura do paciente ou responsável na segunda via da prescrição; "Encaminhamentos", se havia assinatura do paciente ou responsável na segunda via presente; "Termo de Ciência", se havia assinatura do paciente ou responsável na segunda via presente; e "Radiografias", se as imagens estavam viáveis e corretamente acondicionadas.

O método utilizado para a avaliação dos prontuários foi desenvolvido e validado pelo GEOPOL $^{8}$. Ele é fundamentado na classificação do prontuário em 4 situações, representada por 4 cores (vermelha, amarela, azul e verde), com base na gravidade da situação de preenchimento.

A cor vermelha foi atribuída aos prontuários em que tenha faltado alguma assinatura do paciente ou responsável ou alguma informação sobre ele, pertinente ao tratamento ou à identificação, condição considerada mais grave para fins de registro e de produção de provas.

A cor amarela foi usada nos casos em que tenha faltado alguma assinatura do docente ou do aluno responsável pelo tratamento.

A cor azul foi usada nos prontuários que continham falha ou ausência de preenchimento no envelope que acondiciona os prontuários, fato que pode trazer prejuízos de evolução clínica dos pacientes.

A cor verde foi atribuída aos prontuários em que não havia nenhuma correção a ser feita e se encontravam de acordo com o que preconiza a literatura concernente aos parâmetros éticos e legais da documentação odontológica.

Os dados foram processados em planilhas eletrônicas do Microsoft Excel. Os resultados foram apresentados de modo descritivo com valores absolutos e percentuais e foi realizada análise estatística por meio do teste QuiQuadrado $\left(\chi^{2}\right)$ coeficiente de contingência $C$, com auxílio do Software Bioestat 5.3 e considerando um nível de significância de $5 \%$.

$\mathrm{O}$ estudo foi aprovado pelo Comitê de Ética em Pesquisa com Seres Humanos da UEM (CAAE 85891018.8.0000.0104).

\section{RESULTADOS}

A tabela 1 demonstra a condição dos prontuários da COD-UEM nas duas análises. Verificou-se na Análise 2 a diminuição no percentual de prontuários classificados como "vermelho" em relação à Análise $1(\mathrm{p}=0,006)$. Além do mais, na análise 2, houve considerável aumento de prontuários verdes $(\mathrm{p}<0,0001)$.

Tabela 1. Descrição absoluta (n), percentual $(\%)$ e analítica (p) das variáveis relacionadas à Classificação de Risco de prontuários da COD-UEM avaliados antes e após a implantação da Gestão de Risco

\begin{tabular}{|c|c|c|c|c|c|}
\hline \multirow[b]{2}{*}{ CLASSIFICAÇÃO } & \multicolumn{2}{|c|}{ ANÁLISE $1(\mathrm{n}=660)$} & \multicolumn{2}{|c|}{ ANÁLISE $2(n=422)$} & \multirow[b]{2}{*}{$\mathrm{p}$} \\
\hline & $\mathrm{n}$ & $\%$ & $\mathrm{n}$ & $\%$ & \\
\hline Vermelho & 610 & 92,42 & $301 *$ & 71,33 & 0006 \\
\hline Amarelo & 31 & 4,70 & 31 & 7,35 & \\
\hline Azul & 7 & 1,06 & 25 & 5,92 & \\
\hline Verde & 12 & 1,82 & $65^{* *}$ & 15,40 & $<0.0001$ \\
\hline
\end{tabular}

$(*=\mathrm{p}<0,05, * *=\mathrm{p}<0,001)$

A tabela 2 apresentou as variáveis do tratamento. Observou-se na Análise 1 que, a relacionadas à identificação e declaração de ciência variável "nome do paciente" foi preenchida em 
todos os prontuários $(100,0 \%)$, assim como na Análise 2, enquanto a variável "Termo de ciência" se apresentou com maior percentual de preenchimento correto na Análise 2, com resultado significativo em termos estatísticos, em comparação com a Análise $1 \quad(\mathrm{p}=0,002)$. As variáveis "documento" e "assinatura" não apresentaram diferença estatística.

Tabela 2. Descrição absoluta (n), percentual (\%) e analítica (p) das variáveis relacionadas à identificação e declaração de ciência dos pacientes atendidos na COD-UEM com prontuários institucionais avaliados antes e após a implantação da Gestão de Risco

\begin{tabular}{ccccccccccc}
\hline & \multicolumn{3}{c}{ ANÁLISE 1 (n=660) } & \multicolumn{4}{c}{ ANÁLISE 2 (n=422) } \\
& \multicolumn{2}{c}{ Campo } & \multicolumn{2}{c}{ Não preenchido/ } & \multicolumn{2}{c}{ Campo } & Não preenchido/ & \\
& preenchido & \multicolumn{2}{c}{ incompleto ou à lápis } & \multicolumn{2}{c}{ preenchido } & incompleto ou à lápis & \\
VARIÁVEIS & $\mathrm{n}$ & $\%$ & $\mathrm{n}$ & $\%$ & $\mathrm{n}$ & $\%$ & $\mathrm{n}$ & $\%$ & $\mathrm{p}$ \\
\hline Nome & 660 & 100,0 & 0 & 0,0 & 422 & 100,0 & 0 & 0,0 & - \\
Documento & 488 & 73,94 & 172 & 26,06 & 358 & 84,83 & 64 & 15,17 & 0,154 \\
Assinatura & 643 & 97,42 & 17 & 2,58 & 411 & 97,39 & 11 & 2,61 & 0,967 \\
Termo de Ciência & 423 & 64,09 & 237 & 35,91 & $364 *$ & 86,26 & 58 & 13,74 & 0,002 \\
\hline
\end{tabular}

$(*=\mathrm{p}<0,05)$

As variáveis analisadas na tabela 3 se referem à anamnese e aos exames do paciente, bem como ao plano de tratamento e as devidas assinatura no mesmo. Foi possível observar que as variáveis "Anamnese" e "Periograma", embora tivessem um percentual maior de preenchimento correto na Análise 2, não houve significância estatística. Foi observado ainda que, as variáveis "Exame físico" e "Plano de tratamento" obtiveram resultados estatisticamente significativos ( $\mathrm{p}<0,0001)$, assim como a variável "Odontograma" que também teve um percentual de preenchimento significativamente maior na segunda análise $(\mathrm{p}=0,009)$.

Tabela 3. Descrição absoluta (n), percentual (\%) e analítica (p) das variáveis relacionadas à identificação e declaração dos pacientes atendidos na COD-UEM com prontuários institucionais avaliados antes e após a implantação da Gestão de Risco

\begin{tabular}{|c|c|c|c|c|c|c|c|c|c|}
\hline \multirow[b]{3}{*}{ VARIÁVEIS } & \multicolumn{4}{|c|}{ ANÁLISE 1 (n=660) } & \multicolumn{4}{|c|}{ ANÁLISE 2 (n=422) } & \multirow[b]{3}{*}{$\mathrm{p}$} \\
\hline & \multicolumn{2}{|c|}{$\begin{array}{l}\text { Campo } \\
\text { preenchido }\end{array}$} & \multicolumn{2}{|c|}{$\begin{array}{c}\text { Não preenchido/ } \\
\text { incompleto ou à lápis }\end{array}$} & \multicolumn{2}{|c|}{$\begin{array}{l}\text { Campo } \\
\text { preenchido }\end{array}$} & \multicolumn{2}{|c|}{$\begin{array}{c}\text { Não preenchido/ } \\
\text { incompleto ou à lápis }\end{array}$} & \\
\hline & $\mathrm{n}$ & $\%$ & $\mathrm{n}$ & $\%$ & $\mathrm{n}$ & $\%$ & $\mathrm{n}$ & $\%$ & \\
\hline Anamnese & 578 & 87,58 & 82 & 12,42 & 397 & 94,08 & 25 & 5,92 & 0,454 \\
\hline Exame físico & 324 & 49,09 & 336 & 50,91 & 336 & 79,62 & $86^{*}$ & 20,38 & $<0,0001$ \\
\hline Odontograma & 368 & 55,76 & 292 & 44,24 & 306 & 72,51 & $116^{*}$ & 27,49 & 0,009 \\
\hline Periograma & 519 & 78,64 & 141 & 21,36 & 393 & 93,13 & 29 & 6,87 & 0,071 \\
\hline Plano de tratamento & 194 & 29,39 & 466 & 70,61 & 285 & 67,54 & $137 *$ & 32,46 & $<0,0001$ \\
\hline $\begin{array}{c}\text { Assinatura do } \\
\text { estudante }\end{array}$ & 525 & 79,55 & 135 & 20,45 & 312 & 73,93 & 110 & 26,07 & 0,469 \\
\hline $\begin{array}{l}\text { Assinatura do } \\
\text { docente }\end{array}$ & 497 & 75,30 & 163 & 24,70 & 286 & 67,77 & 136 & 32,23 & 0,298 \\
\hline $\begin{array}{l}\text { Assinatura do } \\
\text { paciente }\end{array}$ & 522 & 79,09 & 138 & 20,91 & 289 & 68,48 & 133 & 31,52 & 0,147 \\
\hline
\end{tabular}


A tabela 4 se refere às assinaturas do aluno, do paciente e do docente no relatório de atividades clínicas. Nas variáveis "assinatura do aluno" o percentual da Análise $1(98,18 \%)$ foi maior que o da análise 2 (97,87\%), entretanto nas outras duas variáveis o percentual foi maior na segunda análise. Estatisticamente falando, não houve diferença entre as análises.

Tabela 4. Descrição absoluta (n), percentual (\%) e analítica (p valor) das variáveis relacionadas aos registros de assinaturas nos procedimentos dos pacientes atendidos na COD-UEM com prontuários institucionais avaliados antes e após a implantação da Gestão de Risco

\begin{tabular}{|c|c|c|c|c|c|c|c|c|c|}
\hline \multirow[b]{3}{*}{ VARIÁVEIS } & \multicolumn{4}{|c|}{ ANÁLISE 1 (n=660) } & \multicolumn{4}{|c|}{ ANÁLISE 2 (n=422) } & \multirow[b]{3}{*}{$\mathrm{p}$} \\
\hline & \multicolumn{2}{|c|}{$\begin{array}{c}\text { Campo } \\
\text { preenchido }\end{array}$} & \multicolumn{2}{|c|}{$\begin{array}{l}\text { Não preenchido/ } \\
\text { incompleto ou à } \\
\text { lápis }\end{array}$} & \multicolumn{2}{|c|}{$\begin{array}{c}\text { Campo } \\
\text { preenchido }\end{array}$} & \multicolumn{2}{|c|}{$\begin{array}{l}\text { Não preenchido/ } \\
\text { incompleto ou à } \\
\text { lápis }\end{array}$} & \\
\hline & $\mathrm{n}$ & $\%$ & $\mathrm{n}$ & $\%$ & $\mathrm{n}$ & $\%$ & $\mathrm{n}$ & $\%$ & \\
\hline Assinatura do estudante & 648 & 98,18 & 12 & 1,82 & 413 & 97,87 & 9 & 2,13 & 0,993 \\
\hline Assinatura do docente & 582 & 88,18 & 78 & 11,82 & 391 & 92,65 & 31 & 7,35 & 0,615 \\
\hline Assinatura do paciente & 640 & 96,97 & 20 & 3,03 & 414 & 98,10 & 8 & 1,90 & 0,930 \\
\hline
\end{tabular}

A tabela 5 demonstrou os resultados referentes às variáveis "documentos emitidos ao paciente e exames de imagem". Nesse caso apresentou em valores absolutos e percentuais a ocorrência de documentos presentes e incorretos ou não viáveis. Não houve diferença estatística entre as análises para nenhum dos documentos.

Tabela 5. Descrição absoluta (n), percentual (\%) e analítica (p valor) das variáveis relacionadas aos documentos emitidos ao paciente e exames de imagem pacientes atendidos na COD-UEM com prontuários institucionais avaliados antes e após a implantação da Gestão de Risco

\section{ANÁLISE 1 (n=660) ANÁLISE 2 (n=422)}

Presente e incorreto ou não viável Presente e incorreto ou não viável

\begin{tabular}{cccccc} 
VARIÁVEIS & $\mathrm{n}$ & $\%$ & $\mathrm{n}$ & $\%$ & $\mathrm{p}$ \\
\hline Atestados & 112 & 16,97 & 80 & 18,96 & 0,537 \\
Receitas & 52 & 7,88 & 23 & 5,45 & 0,189 \\
Encaminhamentos & 12 & 1,82 & 3 & 0,71 & 0,217 \\
Radiografias & 98 & 14,85 & 56 & 13,28 & 0,588 \\
\hline
\end{tabular}

Quanto ao protocolo de classificação adotado para os prontuários, pode-se observar que enquanto o percentual de prontuários "Vermelhos" na Análise 1 contabilizava $92 \%$ da amostra (610 prontuários), na Análise 2 esta proporção diminui para $71 \%(n=301)$.
Já no que se diz respeito aos prontuários classificados como "Verde", enquanto na Análise 1 estes somavam apenas $1 \%$ da amostra total, na segunda análise este valor aumentou para $15 \%$.

Tais dados ressaltam $\mathrm{o}$ aumento da preocupação com o preenchimento desses 
documentos e a proteção frente a questionamentos na esfera ética, civil ou criminal da COD-UEM.

\section{DISCUSSÃO}

Segundo Almeida et al. ${ }^{9}$, a identificação completa consiste em nome completo, documento, data de nascimento, nacionalidade, naturalidade, gênero, estado civil, filiação, profissão, endereço residencial e profissional e telefone residencial ou celular. Nos casos de pacientes menores de 18 anos ou incapazes, deve haver também os dados referentes ao responsável legal, bem como suas assinaturas.

Nesta pesquisa documental verificou-se que a variável "Nome" estava presente em todos os prontuários nas duas análises, sendo a única variável a apresentar essa característica. No que se refere a variável "documento" houve uma evolução de quase $10 \%$ na Análise 2 (84\%) quando comparada à anterior $(73,9 \%)$, mas o ideal seria estar presente na totalidade, visto que se trata de um dos dados referentes à identificação completa do paciente.

O Termo de Consentimento Livre e Esclarecido é a ciência e aprovação do paciente a respeito do tratamento a ser realizado, pois nele, o paciente confirma ter conhecimento sobre riscos que o tratamento oferece e declara ter recebido todas as orientações e informações pertinentes ${ }^{10}$, o que trará um respaldo ao profissional no caso de futuros acionamentos jurídicos devido a complicações em procedimentos. Além disso, para o Código de Ética Odontológica constituí infração ética realizar qualquer procedimento sem a assinatura do paciente ou responsável no Termo de Consentimento $^{10}$.

Nesse sentido, foi observado que a variável "Assinatura" do consentimento ao tratamento odontológico estava preenchida em $97 \%$ dos casos mesmo antes da implantação da Gestão de Risco e assim se manteve na Análise 2. Corroborando com o estudo de Costa et al. ${ }^{1}$, onde o resultado obtido foi de $94 \%$ de prontuários assinados, o que mostra a consciência dos alunos quanto à importância deste dado no prontuário do paciente nas duas pesquisas documentais.

A variável "Termo de Ciência" representa um aspecto imprescindível ao bom andamento legal de uma instituição de Ensino ou clínica particular, sendo necessária a presença de duas vias, uma delas entregue ao paciente (ou responsável legal) e a outra anexada ao prontuário $^{12}$. A evolução dessa variável foi demonstrada através do aumento de $22 \%$ da Análise 2 sobre a Análise 1 .

Em um contexto geral, embora ainda existam melhorias necessárias a serem implementadas, verificou-se que, mais de $80 \%$ dos prontuários analisados após a Gestão de Risco estavam corretos no que se diz respeito à identificação geral do paciente.

Através da "anamnese" é possível obter a história médica e odontológica do paciente, bem como sua queixa principal, a história da doença atual, medicamentos que o mesmo faça uso, hábitos e outras informações relevantes ao tratamento $9,13,14$. Houve uma evolução no preenchimento dessa variável após a implantação da Gestão de Risco, de $87 \%$ da Análise 1 para 94\% na Análise 2, aumentando a quantidade de prontuários que abrangem melhor as condições de saúde do paciente para poder realizar o acompanhamento e tratamento ideais para cada caso, considerando suas particularidades dos pacientes por meio dessas informações importantes ${ }^{15,16}$.

Avaliou-se nesse estudo que o percentual de preenchimento correto da variável "Exame Físico" teve um aumento de $30 \%$ após a implantação da Gestão de Risco. Este dado é de grande valia, visto que o estudo de Brandão et al. ${ }^{16}$ demonstra que o exame físico é muito importante para que o profissional possa fazer o diagnóstico, planejar o tratamento, acompanhar e avaliar a evolução de 
cada paciente por meio dos sinais vitais e inspeções intra e extra orais, visto que o $\mathrm{CD}$ tem responsabilidade de identificar anormalidades.

Os dados que se obtém dos exames dos pacientes podem auxiliar também em casos de identificação de vítimas em que não é possível identificá-las por outro método, que não a comparação dos registros documentados com os achados que necessitam ser identificados ${ }^{17,18}$, e nesse sentido pode-se atribuir enorme importância ao Odontograma, que nos achados do presente estudo, estava presente e completo em $55 \%$ dos prontuários analisados em 2017, e na segunda avaliação o percentual chegou a $72 \%$, demonstrando evolução no preenchimento porém não sendo ainda o ideal. Também foi notável a evolução do campo preenchido na variável "Periograma", que mostrou um aumento do percentual em $15 \%$ após a Gestão de Risco ser implantada.

O Plano de Tratamento, segundo Silva et $a l .{ }^{14}$, deve esclarecer o paciente a respeito do que será necessário para a resolução de seu caso, e deve haver opções disponíveis e adequadas para alcançar o objetivo odontológico que satisfaça às necessidades do paciente. Esse plano deve estar claro e compreensível, além de necessitar da aprovação do paciente ou de seu responsável legal.

No presente estudo, foi averiguado que a variável "Plano de Tratamento" estava presente e correta em apenas em 67\% dos prontuários avaliados na Análise 2, demonstrando uma considerável evolução percentual e analítica, porém do ponto de vista legal ainda não é o desejável. Quanto às assinaturas no plano de tratamento, para Silva et al. ${ }^{14}$, elas significam ciência e autorização para que seja executada pelo profissional a alternativa escolhida pelo paciente $\mathrm{e}$ entendida como a ideal para o caso.

Contrariando o fato que sugere a importância das assinaturas no Plano de Tratamento, a variável "assinatura do paciente" apresentou um percentual de $68 \%$ de preenchimento, sofrendo diminuição quando comparada à Análise anterior à Gestão de Risco, assim como as assinaturas do docente e do aluno responsável que também se mostraram com preenchimento mais deficiente na Análise 2.

Esse resultado pode ser justificado pelo fato de que na Análise 2 foram excluídos prontuários nos quais não havia registros de procedimentos após a Análise 1. Muitos desses prontuários estavam com todas as devidas assinaturas no Plano de Tratamento, e a partir do momento em que eles foram excluídos da segunda amostra, o percentual de ocorrência dessa variável ficou menor.

Na prática clínica é imprescindível que os procedimentos executados sejam descritos de forma completa e clara no prontuário, além de sempre ser necessária a assinatura do paciente em questão ou de seu responsável legal, para conferir validade ao exposto e aumentar a garantia de proteção jurídica $^{13}$.

As assinaturas nos procedimentos executados foram variáveis que se encontraram com alto índice de preenchimento nas duas análises. Embora o percentual de preenchimento da variável "Assinatura do Estudante" sofreu um decréscimo na Análise 2, por outro lado, ainda foi de $97 \%$, representando um bom percentual e dentro do esperado e aceitável as variáveis "Assinatura do docente" e "Assinatura do paciente" tiveram um resultado positivo, visto que mais de $92 \%$ dos prontuários continham a assinatura do docente, e quanto à assinatura do paciente, mais de $98 \%$ dos prontuários condiziam com o ideal.

Os atestados e receitas foram outras variáveis averiguadas, visto que estes documentos precisam estar preenchidos em duas vias (exceto receitas especiais, que são elaboradas em três vias), devendo a segunda ser assinada pelo paciente, ou responsável legal, e anexada ao prontuário. Deste modo, foi encontrado neste estudo, como a única variável que não melhorou na segunda análise, o 
porcentual de assinaturas na segunda via dos atestados, subindo de $17 \%$ para $19 \%$, demonstrando uma menor atenção com essa assinatura e evidenciando a necessidade de maior controle dos docentes perante a este fato. Já o esquecimento da solicitação de assinaturas nas segundas vias das receitas diminuiu $2 \%$ na Análise 2.

A variável "Encaminhamento" apresentou um ótimo percentual pois menos de $1 \%$ desse tipo de documento estava incorreto, apresentando- se sem assinatura do paciente. Garantindo assim, que o paciente confirme que foi encaminhado e a instituição possa possibilitar o fluxo de referência e contra referência adequadamente das Redes de Atenção a Saúde que melhoram o acesso e a qualidade da atenção prestada ${ }^{19}$.

As radiografias são consideradas um dos mais importantes exames complementares, pois avaliam a condição anterior ao tratamento e após os procedimentos realizados, servindo como um importante meio de proteção legal. Para tanto, é necessário que a estejam bem processadas $\mathrm{e}$ armazenadas de maneira correta para manter a qualidade ao longo do tempo ${ }^{9}$, devido a isso, este foi outro quesito analisado durante a gestão de risco. Observou-se na primeira análise, $14 \%$ das radiografias consideradas inviáveis, já na segunda esse percentual foi de $13 \%$, sendo possível e necessário ainda, melhorar esse quesito. Visto que o raio $\mathrm{X}$ é o exame complementar mais utilizado pelo $\mathrm{CD}$ e necessita produzir os efeitos legais desejados seja no âmbito ético, administrativos, judiciais ou pericial ${ }^{4}$.

Para Fernandes ${ }^{20}$, considerando reuniões do SBPqO e artigos na plataforma Scielo nos anos de 2013 e 2014, ainda faltavam estudos relacionados à elaboração e manutenção de prontuários, demonstrando dessa forma a necessidade de maior atenção ao tema e desenvolvimento de mais pesquisas científicas a respeito.

É extremamente importante que o acadêmico de Odontologia comece a se conscientizar desde a graduação a respeito da necessidade da elaboração de uma boa documentação, pois esse é o momento em que ele pode aprender que um prontuário completo é seu aliado profissional ${ }^{21}$. As instituições de ensino são responsáveis pela conscientização da importância de uma documentação odontológica bem elaborada e completa, já que é na formação que os futuros profissionais agregam hábitos para toda vida ${ }^{4}$.

Os resultados encontrados demonstram que a gestão de risco é de extrema importância para a melhora dos indicadores, conforme já verificado. Entretanto, esta ação isolada não é capaz de assegurar o sucesso documental, sendo de extrema importância a conscientização e colaboração de todo o corpo docente, dos discentes e servidores técnicos quanto à importância do preenchimento correto e completo do prontuário odontológico. Além do mais, a análise documental deve ser um processo dinâmico, gerando feedback para a instituição ter melhores condições de avaliação e planejamento.

\section{CONCLUSÃO}

A gestão de risco documental proposta refletiu em evolução no preenchimento dos prontuários clínicos. Houve redução significativa de documentos contendo falhas e aumento da documentação ética e legalmente segura. Muito contribuiu para isto a melhoria significativa de preenchimento do termo de ciência, do exame físico, do plano de tratamento e do odontograma. Foi possível perceber que não houve avanço para todos os campos da documentação, fato este que traz a reflexão de que a gestão documental deve ser dinâmica e contínua, pautando-se nos ajustes documentais e nos processos de conscientização.

\section{ABSTRACT \\ Evolution analysis of institutional clinical record filling after the implementation of risk}




\section{management}

This documentary, longitudinal and quantitative study was carried out in order to evaluate the documentary evolution institutional clinical records after the implementation of risk management. In 2017, Legal Dentistry and Professional Orientation Study Group (GEOPOL), from State University of Maringá (UEM) in order to improve document production and reduce risks. The present study considered 660 records analyzed by GEOPOL (Analysis 1) from March 2017 to April 2018. Between May and September 2018, 422 records were evaluated (Analysis 2), after the implementation of risk management. Both analyzes were based on an exclusive checklist, developed by GEOPOL for this purpose. Data were processed in electronic spreadsheets and results were presented in a descriptive and analytical way. The results showed that, in general, the variables presented a better filling in Analysis 2, demonstrating effectiveness in GEOPOL management processes. It was concluded that there was an evolution in documentary filling of clinical records after the implementation of risk management.

Descriptors: Legal Dentistry. Documentation. Dental Ethics. Risk Management.

\section{REFERÊNCIAS}

1. Costa SM, Braga SL, Abreu MHNG, Bonan PRF. Questões éticas e legais no preenchimento das fichas clínicas odontológicas. RGO. 2009; 57(2): 211-6.

2. Coltri MV, Silva RHA. Prontuário do paciente: comentários à Lei ${ }^{\circ} 13.787 / 2018$. Rev Bras Odontol Leg RBOL. 2019;6(2):89105.

3. Oliveira DL, Yarid SD. Prontuário odontológico sob a ótica de discentes de Odontologia. Rev Odontol da UNESP. 2014; 43(3): 158-64.

4. Amorim HPL, Marmol SLP, Cerqueira N, Silva MLCA, Silva UA. A importância do preenchimento adequado dos prontuários para evitar processos em Odontologia. Arq Odontol 2016; 52(1): 32-7.

5. Onesti A. Odontologia Defensiva e Prática Clínica de Baixo Risco. [Tese]. São Paulo: Faculdade de Odontologia de São Paulo; 2010.

6. Mota JMS, Araújo ME, Carres FCA, LopezCapp TT, Biazevic MGH, Michel Crosato E. Personal and professional profile, education, and perception of management and entrepreneurship of Dentistry students. Rev ABENO. 2018; 18(4): 85-94

7. Rocha IS, Andrade G, Lolli LF, Pereira MC. Avaliação do preenchimento de prontuários odontológicos de uma instituição de ensino superior frente aos aspectos éticos e legais [Monografia]. Maringá: Universidade Estadual de Maringá, 2013.

8. Lolli LF, Silva MC, Campos FNL, Mathias AP, Nemer MRM. Odontologia Defensiva e educação permanente: gestão de prontuários contribuindo na formação de cirurgiõesdentistas com responsabilidade profissional. Rev ABENO. 2019; 19(4):113-22.

9. Almeida CAP, Zimmermann RD, Cerveira JGU, Julivaldo FSN. Prontuário odontológico: uma orientação para o cumprimento da exigência contida no inciso VIII do art. $5^{\circ}$ do Código de Ética Odontológica [Acesso em 10 out. 2018]. Disponível em: http://www.cromg.org.br/ normatizacoes/prontuario_odontologico.pdf

10. Paranhos LR, Guedes TMP, Jóias FC, Scanavini MA. Orientações legais aos ortodontistas: elaboração de Contrato de Prestação de Serviços Odontológicos e Consentimento Esclarecido - Parte II. Ortodontia SPO. 2009; 42 (3):237-43.

11. Conselho Federal De Odontologia. Código de Ética Odontológica; 2012 [Acesso em 5 out. 2018]. Disponível em: http://cfo. 
org.br/wpcontent/uploads/2009/09/codigo_ etica.pdf

12. Costa SM. Pesquisa envolvendo seres humanos. Abordagem bioética. Rev CROMG. 2002; 8(3): 221-6.

13. Silva RHA. Orientação Profissional para o Cirurgião Dentista: Ética e Legislação. São Paulo: Santos; 2010.

14. Silva RF, Prado MM, Rodrigues LG, Pícoli FF, Franco A. A Importância Ético-Legal e Significado das Assinaturas do Paciente no Prontuário Odontológico. Rev Bras Odontol Leg RBOL. 2016; 3(1): 70-83.

15. Peruchi CTR, Souza AP, Hidalgo MM, Terada RSS, Peixoto IF, Bispo CGC. A clínica ampliada na Odontologia: avaliação do trabalho clínico onde o ensino acontece. Rev Assoc Paul Cir Dent. 2015; 69(2):169-77.

16. Brandão BA, Cortez DL, Loureiro DL, Moraes GR, Bréda MA, Fernandades DC. Importância de um exame físico adequado para o atendimento odontológico. Cad Grad Ciênc Bio Saúde Unit. 2018; 5(1):77-87.

17. Paranhos LR, Caldas JCF, Iwashita AR, Scanavini MA, Paschini RC. A importância do prontuário odontológico nas perícias de identificação humana. RFO. 2009; 14(1): 14-7.

18. Silva M, Paula FJ, Zimmermann RD.
Deontologia Odontológica. Ética e Legislação. São Paulo: Santos; 2011.

19. Souza GC, Lopes MLDS, Roncalli AG, Medeiros-Junior A, Clara-Costa IC. Referência e contra referência em saúde bucal: regulação do acesso aos centros de especialidades odontológicas. Rev Salud Pública. 2015; 17 (3): 416-28.

20. Fernandes RCD. Quantitativo de publicações científicas contemplando a importância do adequado preenchimento, guarda e conservação dos prontuários odontológicos nos anos de 2013 e 2014 [Monografia]. Araruna: Universidade Estadual da Paraíba; 2015.

21. Benedicto EN, Lages LHR, Oliveira OF, Silva RHA, Paranhos LR. A importância da correta elaboração do prontuário odontológico. Odonto. 2010; 18 (36): 41-50.

\section{Correspondência para:}

Luiz Fernando Lolli

e-mail: profdrluizfernando@gmail.com

Universidade Estadual de Maringá

Av. Mandacaru, 1550 - Centro

87080-000 Maringá/PR 